\title{
ALGUNAS REFLEXIONES PARA LA PREHISTORIA Y ARQUEOLOGÍA: LAS MUJERES EN LA CONSTRUCCIÓN DE LA HISTORIA ${ }^{1}$
}

\section{SOME THOUGHTS FOR PREHISTORY AND ARCHAEOLOGY: WOMEN IN THE CONSTRUCTION OF HISTORY}

\author{
por
}

OLGA SÁNCHEZ LIRANZO

(De nuevo a mi maestro y amigo Enrique Vallespi ${ }^{2}$ )

RESUMEN

ABSTRACT

Palabras claves

Key words
En este trabajo exponemos algunas reflexiones de carácter teórico-metodológico aparecidas hace más de una década por parte de la "Nueva Historia de las Mujeres", y que aún están por plantear en la ciencia arqueológica de nuestra región.

In this work present some theoretic and methodologic reflections from a decade ago referred to the "New Women's Studies", and we aren't bring up yet in the archaeology science of the our region.

Prehistoria, arqueología, mujeres, historiografía, Nueva Historia de las Mujeres, feminismo, androcentrismo, género, vida cotidiana.

Prehistory, archaeology, women, historiography, New Women's History, feminism, androcentrism, gender, daily life.

1. Este artículo tiene su origen en la conferencia "Las mujeres en la construcción de la Historia" del ciclo de Historia de Género, celebrada en Porcuna (Jaén) en septiembre de 2000.

2. Estoy segura que todas y todos coincidirán conmigo que éste es un merecido homenaje que tardaba en llegar, porque en estos tiempos no sólo escasean paleolitistas de la talla de Enrique Vallespí, sino personas con su calidad humana. A nivel personal he de reconocer que le debo no sólo mi conocimiento y mi interés por las industrias líticas prehistóricas, sino sobre todo el espíritu renovador y aperturista que siempre me ha transmitido en el estudio de la prehistoria. Esta es la razón por la que he considerado adecuado dedicarle este trabajo que intenta poner de relieve la impronta reflexiva de mi sabio y entrañable maestro y amigo Enrique Vallespí, de la que nunca espero alejarme. 


\section{INTRODUCCIÓN}

Hablar sobre la pre-historia y las mujeres no es una tarea fácil, no sólo por la amplitud y diversidad de temas que se podrían tratar, sino por la variedad de puntos de vistas existentes sobre esta cuestión. Por ello, pretendo que este artículo se convierta en una pequeña introducción que nos sirva como punto de partida para reflexionar sobre la prehistoria y la historia y sobre nuestro papel en ella, como investigadoras/es y como sujetos históricos. De esta forma podremos no sólo cuestionar la propia historia, sino proponer alternativas a la prehistoria y arqueología existente, que recoja y recupere nuestras vivencias y experiencias a lo largo del tiempo.

Tal vez, sea importante que se tenga en cuenta que todas estas reflexiones tienen su origen en un estudio historiográfico y teórico que realicé desde una porción de la historia: la prehistoria ${ }^{3}$. En este trabajo pretendíamos analizar la "ausencia de las mujeres" como objetos de investigación (como mujeres sujetos de la prehistoria) (Sánchez Liranzo 1999). Para dicha investigación teníamos claro, en primer lugar, que las mujeres prehistóricas y sus restos arqueológicos, no debían ser ni más ni menos numerosos, ni más ni menos visibles que los hombres prehistóricos y sus restos. La cuestión, por tanto, no podía centrarse en la ausencia de las mujeres en el registro arqueológico, sino en que esta ausencia era "producto" de las personas que desarrollaban esas investigaciones: los arqueólogos y arqueólogas. Es decir, el problema se encontraba en los presupuestos teóricos e ideológicos, en las ideas previas de los investigadores e investigadoras que "recopilaban" y "seleccionaban" aquello que consideraban relevante para la construcción de la historia. En este sentido, la arqueología sólo daba respuestas a las preguntas que se hacían previamente. Se evidenciaba que una cosa era la "historia objetiva" (la realidad) y otra la "historia producida" (el conocimiento de esa realidad) (Bate 1998) y que ambas no siempre coincidían. Descubrimos, entonces, que teníamos que comenzar nuestro estudio adentrándonos en el mundo de las valoraciones y los presupuestos teóricos, lo que se denomina "área valorativa", es decir, una serie de juicios éticos e ideológicos que vienen a determinar en último extremo para qué investigamos (Gándara 1993).

En segundo lugar, éramos conscientes que el pensamiento que impregna la prehistoria y las ciencias sociales en general, era un pensamiento etnocéntrico, porque tomaba la cultura occidental como base para evaluar, analizar y juzgar otras culturas; egocéntrico, porque situaba al hombre blanco, adulto, de clase media, occidental y a sus valores como centro de la investigación histórica, despreciando todo lo que resulta ser ajeno a él; y dicotómico, porque todo lo que se considera "ser diferente a algo" se asume al final como "opuesto a ese algo", y estableciéndose así unas relaciones desiguales y jerárquicas entre ambos (Sánchez 1991). Esta fue la herencia que la ciencia moderna del siglo XIX dejó en nuestro pensamiento, consolidándose a partir de ella la tradicional y asumida asociación de lo femenino con el sentimiento, la subjetividad, lo doméstico, lo privado, la reproducción, la naturaleza,...; y lo masculino con la razón, la objetividad, lo público, la producción, la cultura,...

De tal forma que uno de los objetivos que nos marcamos era descubrir el carácter ideológico que escondía el uso de las dualidades en nuestra disciplina. Es decir, estudiar qué ideas hay detrás de la división que siempre establecemos entre los sexos, sus actividades, sus comportamientos, sus espacios, sus caracteres,... en la historia. Conseguimos así poner de manifiesto los prejuicios que contaminan las descripciones, interpretaciones y explicaciones históricas de los investigadores e investigadoras que nos hacían pensar que la desigualdad social de sexo y género era algo innato, que siempre ha estado vinculado a la "naturaleza" humana. El estudio historiográfico, la historia de la historia y la prehistoria y arqueología, nos permitían descubrir los prejuicios machistas y las ausencias femeninas que la ciencia histórica ha heredado y asumido sin reflexión. Se trataba de analizar cómo hemos producido y reproducido la información que en la actualidad manejamos

3. La ausencia de las mujeres en la construcción de la historia: el ejemplo de la prehistoria andaluza. Una ciencia social que reproduce el discurso histórico androcéntrico. Tesis de licenciatura inédita. Sevilla, 1998. 
los/as prehistoriadores/as y/o arqueólogos/as (Sánchez Liranzo 1999; 2000; Vera Fernández y Sánchez Liranzo 2000).

Hay que tener en cuenta que toda nueva investigación histórica parte de un conjunto de información que ha sido producida anteriormente por otros/as investigadores/as y que a la vez ésta nueva investigación servirá de base para otros/as investigadores/as futuros/as. De manera, que podemos decir que el conocimiento histórico es acumulativo y está en continua y constante construcción, nunca está acabado. Para entender este aspecto debemos ser conscientes que los/as pre-historiadores/as no somos objetivos/as, es decir que estamos condicionados/as no sólo por el nivel de conocimiento que hemos heredado, sino por nuestra situación económica, social, política e ideológica que influye en nuestra manera de pensar y nuestro sistema de valores del que anteriormente hemos hablado.

El prehistoriador o prehistoriadora (y/o arqueólogo/a) para comprender el pasado va a seleccionar aquellos "hechos históricos" que piensa que son significativos para explicar el desarrollo social. Pero lo que considera importante de lo que no lo es depende, en última instancia, de su marco teórico, de su objetivo investigativo, de su sistema de valores,... En este sentido, el sistema de dualidades que jerarquiza a hombres y mujeres marcará en gran medida qué hechos tiene que seleccionar. Lo que estamos proponiendo es pongamos sobre la mesa el conjunto de valores éticos e ideológicos que guían el conocimiento de la prehistoria andaluza. Desde nuestro punto de vista, éste análisis es esencial, ya que aquí está la clave de porqué las mujeres hemos sido ignoradas en la historia como sujetos de la historia (y prehistoria) y, por tanto, como objeto de conocimiento (Ramos 1990: 189). De hecho, uno de los problemas es que se ha pretendido ocultar el área valorativa, así la historia aparecía como una forma de conocimiento que reflejaba cómo fueron "verdaderamente" las sociedades pasadas. Se imponía una visión universal del mundo, como si esta historia (y prehistoria) se correspondiese clara y objetivamente con la realidad, sin reconocer humildemente que una cosa es la "realidad" histórica y otra su "conocimiento".

\section{ANDROCENTRISMO}

Cuando hablamos de androcentrismo en la prehistoria nos referimos al punto de vista que sitúa al "hombre" en el centro del conocimiento prehistórico. Con este concepto se intenta caracterizar aquella historia (o prehistoria) que considera relevante todo lo que tradicionalmente se asocia al hombre (a sus actividades y comportamientos), por considerarlo lógicamente superior y opuesto a las mujeres. Decimos entonces que la historia es androcéntrica cuando las mujeres están totalmente ausentes en la construcción histórica; o bien cuando, se ofrece una imagen distorsionada de ellas y se les da un papel secundario. La cuestión está lógicamente en que este aspecto de la historia (el androcentrismo) se oculta bajo la etiqueta de la "historia universal" o en nuestro caso concreto de "prehistoria universal", a pesar de que se nos intente convencer que la palabra "hombre" se usa como genérico para referirse tanto a los hombres como a las mujeres, de hecho veremos como el contenido tampoco responde al de una verdadera "Historia Universal".

En nuestra ciencia, una revisión crítica y autocrítica ausente arrastra estos contenidos androcéntricos (y por tanto excluyentes) como herencia de investigadores/as anteriores, transmitiéndolos, sin ser conscientes de ello, a través de la docencia, la elaboración bibliográfica, los museos y otros mecanismos de difusión, proyectándose directa e indirectamente sobre la sociedad.

$\mathrm{El}$ androcentrismo se empieza a descubrir cuando comenzamos a preguntarnos: ¿qué dice la historia (y la prehistoria) de las mujeres?, ¿qué dice la historia de los hombres?, ¿habla de todos los hombres?, ¿de qué hombres concretamente habla? Responder a estas preguntas nos ayuda a resolver otras cómo ¿a quién se refiere entonces la historia cuando supuestamente usa el genérico "hombre"?, ¿a los hombres? 
¿a todos los hombres?, ¿a las mujeres?, ¿a todas las mujeres?, ¿son todos los aspectos históricos seleccionados referidos por igual a unos y a otras? Todas estas cuestiones nos ayudan a resolver la pregunta clave: ¿Quién es el verdadero protagonista y sujeto de esa "pre-historia universal"?(Moreno 1987) ${ }^{4}$.

Analicemos de forma más concreta qué es lo que dice la historia en general sobre las mujeres. Sobre esta cuestión encontramos fundamentalmente dos respuestas. La primera de ellas, la más común, es que no se diga "nada" de las mujeres, porque todo lo que se cuenta está referido al hombre y sus actividades, aunque se habla de "humanidad", "hombre" (como genérico) o "ser humano". Este ha sido en general el caso de nuestra disciplina la prehistoria.

La otra posibilidad es que se diga "algo", aunque casi siempre es para referirse a cómo los/as investigadores/as ven o quieren ver a las mujeres prehistóricas, adscribiéndoles ya desde el principio un papel secundario o inferior respecto de los hombres (prehistóricos). Dentro de este grupo encontramos distintas opciones. Por un lado están las mujeres anónimas, siempre asociadas a la naturaleza, reproducción, espacio doméstico, cocina,... muy común en las reconstrucciones prehistóricas de nuestra región. Por otro, mujeres concretas referidas obviamente a momentos más recientes, históricos ${ }^{5}$.

Pero ¿y de los hombres? ¿qué dice la Historia (y la prehistoria) de ellos? ¿habla de todos? Ya hemos comentado que la historia (y también la prehistoria) es androcéntrica, es decir tiene como objeto de estudio y conocimiento a los hombres, sin embargo nos equivocaríamos si afirmásemos que habla de todos los hombres. Por tanto, no podemos decir que esa prehistoria universal de la que todos los investigadores e investigadoras, en mayor o menor medida, participamos sea una prehistoria "universal" de los hombres porque:

1) Excluir a las mujeres de esta historia es ignorar las relaciones sociales entre hombres y mujeres; y una historia de los hombres que no tenga en cuenta su relación con las mujeres es una historia "incompleta".

2) Tampoco se puede afirmar que esta historia sea la historia de "todos" los hombres, porque el hombre verdaderamente protagonista de esta historia es uno muy concreto: un hombre de raza blanca, adulto, occidental, de clase media,... con una serie de valores asociados con el dominio, la explotación, el poder, la competitividad... Lo que Amparo Moreno ha llamado "el arquetipo viril protagonista de la historia" (1987). Lógicamente este modelo de hombre excluye a gran parte de la población masculina.

3) Finalmente, esta pretendida historia universal de los hombres no es tan universal, porque muestra tan sólo una faceta de la vida de ese hombre blanco, adulto, occidental, aquella faceta que le vincula con la esfera pública y con el modelo de masculinidad que se quiere fomentar. Lejos queda una historia que dé protagonismo a los aspectos de la vida cotidiana de ese hombre.

La arqueología tiene diversos mecanismos de difusión del conocimiento histórico y arqueológico para extenderse y ramificarse sobre la sociedad: los museos, parques arqueológicos, libros de textos, exposiciones, medios de comunicación, etc. En todos ellos se realiza una "selección" del conocimiento arqueológico e histórico que desde la academia y otras instituciones se produce, bien por parte de los/as mismos/as arqueólogos/as o bien por otros/as profesionales relacionados con el campo de la educación. Pero no podemos olvidar que esta "selección" a menudo (consciente o inconsciente) viene a coincidir con los intereses de un grupo concreto, del cual las mujeres no son precisamente las beneficiadas. De hecho, en estos medios de difusión histórica y arqueológica encontramos una selección de contenidos, lenguaje, ilustraciones,...

4. A pesar de que estas reflexiones fueron realizadas hace ya más de una década por distintas historiadoras, apenas se han exportado a la prehistoria.

5. En este caso tenemos aquellas que resaltan por tener un comportamiento masculino y por ello son valoradas como escritoras, reinas, guerreras,...; las que destacan por considerárseles modelos de "virtudes" femeninas que debe imitarse como: esposas, madres, monjas, santas,...; o modelos de mujeres que no hay que seguir, como brujas, prostitutas, curanderas,... (Morant 1997: 8). 
claramente androcéntricos (como son la discriminación lingüística, estereotipos sexuales, exclusión, desequilibrio y selectividad, fragmentación de la realidad), transmitiendo así determinadas pautas de comportamientos y caracteres en función de los sexos y/o géneros.

En definitiva, en la actualidad los/as arqueólogos/as dedicados/as a la investigación, docencia, gestión, conservación, difusión venimos a reproducir sin crítica los mismos protagonistas (hombres adultos blancos occidentales de clase media), las mismas actividades, los mismos valores,... Legitimando un discurso histórico, con el cual no estamos de acuerdo, y que se corresponde con la "cultura del poder", que pertenece a un colectivo excluyente que considera que sus valores son universales y superiores.

\section{HISTORIOGRAFÍA DE LA HISTORIA (Y PREHISTORIA) DE LAS MUJERES}

El descubrimiento y denuncia de la ausencia de las mujeres como sujetos de la historia ha coincidido, por un lado, con el desarrollo de los movimientos feministas de los años 60 y los 70; y por otro, con la incorporación de las mujeres como historiadoras (y prehistoriadoras).

En un primer momento, la ausencia de las mujeres como sujetos históricos se intentó resolver "integrando" o "añadiendo" mujeres a la historia. Con esta medida se intentaba demostrar que había mujeres que habían participado en actividades laborales, en el gobierno, en el arte, en la literatura,... A partir de aquí se elaboraron trabajos e investigaciones que pretendían recuperar parte de nuestra historia para demostrar que nosotras también éramos importantes para el desarrollo de la sociedad.

En los años 70 la llamada Historia Social recupera la historia de aquellos colectivos que habían sido olvidados: las minorías y los/as oprimidos/as. Esta nueva corriente de la historia abrió enormes posibilidades para el nacimiento de la llamada "Nueva Historia de las Mujeres". Gracias al desarrollo de los estudios de demografía histórica, parentesco, familia, matrimonio, sexualidad, vida privada, cultura popular,... comenzó a darse un mayor protagonismo a las mujeres, sobre todo en aspectos que siempre estuvieron relacionados con ellas en su calidad de madre, esposa y ama de casa.

Pero la "Nueva Historia de las Mujeres" intentaba cambiar el enfoque "victimista" que hasta entonces venían adoptando los estudios de la historia de las mujeres. Querían construir una historia de las mujeres en las que ellas fuesen los sujetos protagonistas, escapando de una historia androcéntrica que las ocultaba. Para ello fue necesario convencerse de que su aportación al desarrollo social era más que evidente y que, por tanto, las mujeres no podían considerarse ni "inferiores" ni "secundarias" con respecto a los hombres. Asumir esto significaba que a partir de ahora las mujeres iban a reconocerse así mismas como sujetos protagonistas de la historia, pasando a continuación a convertirse en el objeto de conocimiento de la historia de las mujeres y en una parte fundamental de la historia universal. Este hecho, generará importantes modificaciones teóricas y metodológicas en la ciencia histórica y en la prehistoria que aún hoy estamos por asumir.

El nuevo sujeto histórico (y por tanto también prehistórico) introduce un importante cambio a nivel "teórico". Una nueva forma de percibir la historia (y prehistoria), desde la mirada femenina, supone modificar los conceptos, las categorías y los paradigmas (o los modelos) que la historia (y prehistoria) tradicional usa; pero también, significa crear otros nuevos que recojan aquellos aspectos que antes no le interesaban como las relaciones entre los género, la vida cotidiana, las actividades domésticas, la reproducción,... Así las historiadoras defendían que ahora esta Nueva Historia de las Mujeres no podía tratarse como una temática más dentro de los capítulos de la historia social, sino que debía integrase como parte de la totalidad histórica, ya que afectaba a todos y cada uno de los temas de la historia social.

Lógicamente, un nuevo objeto de conocimiento necesita renovar las formas de acceder al conocimiento, es decir desarrollar nuevas metodologías y transformar las existentes. Por una parte, urgía realizar una nueva 
lectura de las metodologías y las fuentes históricas tradicionales, ya que la mirada y el punto de vista habían sido cambiados radicalmente. Y por otra, era necesario buscar nuevas fuentes de información para una historia más completa (y, por ello, más universal) como son la historia oral, fotografía, diario, cine, arte, cotidianidad,... Es en este punto donde se encuentra hoy la Nueva Historia de las Mujeres.

Desgraciadamente la prehistoria andaluza no sólo no ha asumido aún estas reflexiones, sino que en actualidad gran parte de la comunidad científica las sigue rechazando. A menudo se olvida que dichas cuestiones no incumben únicamente a aquellas/os investigadoras/es que trabajan desde la arqueología del género y/o arqueología feminista, sino también a todas aquellas personas que pretenden aproximarse a la realidad histórica. Es decir, se ignora que dichas reflexiones tienen una carácter meramente "científico", y no sólo "ético".

\section{IV.ALGUNAS PROPUESTAS PARA EL DESARROLLO DE UNA HISTORIA Y PREHISTORIA MÁS "UNIVERSAL"}

Vamos a exponer de forma sintética algunas de las propuestas teóricas y metodológicas de la Nueva Historia de las Mujeres que pueden ser de interés para comenzar a realizar ese despegue que la prehistoria y la arqueología andaluza aún tiene pendiente.

\section{Las mujeres como sujetos prehistóricos e históricos.}

Nuestro objetivo no consiste en otorgar a las mujeres un estatus especial respecto de los hombres. Se trata, ni más ni menos, de que se incorporen como sujetos históricos y por tanto como objeto de nuestro conocimiento prehistórico con todas las consecuencias teóricas y metodológicas que ello supone. Es decir, es importante reconocer que ellas, tanto como los hombres, han contribuido al progreso y desarrollo de la sociedad. De hecho, a lo largo de la historia las mujeres han participado activamente tanto en la producción como en la reproducción humana, es decir hemos colaborado con nuestro trabajo al desarrollo histórico (Martínez 1996):

— Hemos trabajado en las denominadas "actividades domésticas" que integran multitud de labores como cocinar, alimentar a los niños y los enfermos, lavar la ropa, fregar, coser, educar a los hijos e hijas..., actividades que han ido cambiando y modificándose según el contexto histórico en el que se desenvolvían.

- También encontramos mujeres trabajando como campesinas, tejedoras, artesanas, hilanderas, panaderas, criadas, vendedoras, curanderas, matronas, brujas,... e incluso algunas de forma excepcional han podido ser reinas, escritoras, pintoras,... Afortunadamente en la actualidad el abanico de posibilidades se ha abierto considerablemente.

- Pero las actividades que se han considerado más "femeninas" de todas han sido aquellas relacionadas con la reproducción biológica o maternidad, es decir gestar, parir y amamantar. Estas actividades aunque están directamente relacionadas con la "naturaleza humana", no se nos puede olvidar que están reguladas y condicionadas socialmente. El trabajo reproductivo de las mujeres cuyo resultado es dar vida a otros seres humanos casi siempre ha sido poco valorado social y económicamente, de hecho no se reconoce como un "trabajo", sino más bien como "algo" que las mujeres tienen que hacer obligatoriamente por "naturaleza" (Tabet 1986).

En definitiva, las mujeres siempre hemos trabajado a lo largo de la historia en distintas tareas productivas, y siempre en las reproductivas, otra cosa muy distinta es el escaso valor social que estas actividades tienen 
en cada contexto histórico. Podemos decir entonces que "no es que las mujeres hagamos cosas poco importantes, sino que formamos parte de una sociedad que cataloga como poco importante cualquier cosa que hagan las mujeres"(Juliano 1992). Esta reflexión es desde nuestro punto de vista fundamental porque significa que los/as prehistoriadores/as como agentes sociales debemos ser conscientes que nuestras definiciones (como por ejemplo "trabajo") están claramente determinadas por el contexto socio-histórico en el que vivimos. Entender esto nos ayuda a escapar de los prejuicios androcéntricos inherentes en el concepto de trabajo que empleamos y comprender porqué a menudo excluimos determinadas actividades que son realizadas por las mujeres a lo largo de la historia.

\section{La historia de las mujeres: una experiencia histórica común}

El hecho de que prácticamente todas las mujeres hayan vivido a lo largo de la historia bajo unas condiciones de subordinación y explotación, supone que debamos aceptar que casi todas, bajo distintas circunstancias, han tenido una experiencia histórica común. Somos conscientes que no todas las experiencias han sido iguales, entre otros motivos porque en una sociedad no actúan aisladas y autónomamente las relaciones de género y/o sexo, sino que hay que tener en cuenta todo un entramado de relaciones sociales que van a marcar diferencias muy notables dentro del colectivo de mujeres, como son las clases sociales, la edad, la nacionalidad, la etnia, la religión, la raza,... Pero, dentro de la singularidad histórica que supone la experiencia común de las mujeres, hay que respetar la marca de multiplicidad de experiencias femeninas directamente relacionadas con el contexto socio-histórico en el que viven (Martínez 1996: 9).

Hemos comentado cómo la historia y/o prehistoria de los hombres (tradicionalmente llamada "Historia Universal") no es equivalente a una historia de las mujeres, aunque tampoco es equivalente a la historia de todos los hombres. Por tanto, la propuesta de la Nueva Historia de las Mujeres consiste en construir una historia de las mujeres que recupere sus experiencias y vivencias que hasta ahora habían quedado silenciadas. Sin embargo, para aquellos y aquellas que no acaban de "confiar" del todo en las pretensiones feministas o de la Nueva Historia de las Mujeres, hemos de aclarar que nuestro objetivo no es otro que recuperar nuestra memoria histórica y aproximarnos todo lo posible a la realidad histórica o prehistórica, y no hacer una historia de mujeres sin hombres.

Sin embargo, recuperar la prehistoria o historia de las mujeres e integrarla en la prehistoria o historia tradicional, con todas las transformaciones que ello conlleva, no consiste sólo en hacer una historia de la esfera "privada" o "doméstica" que tan abandonada ha sido por otros/as investigadores/as, sino que debe ser una historia que recupere cuestiones sobre cómo las mujeres han vivido la familia, el trabajo, la sexualidad, la democracia, el ámbito público, su propio cuerpo, la comida, la cultura, su relación con los hombres, su relación con otras mujeres, ... y que no sólo analice a las mujeres desde una perspectiva de marginación y explotación, sino también desde su resistencia y lucha contra esa situación (Martínez 1996: 9).

\section{Eliminación de las dicotomías}

Ya hemos advertido que uno de los elementos más importantes para recuperar nuestra historia consiste en eliminar las dicotomías tradicionales hombre-mujer y sus implicaciones en la división de actividades, caracteres, comportamientos, valores,... según los sexos y géneros.

Una asociación muy común es la que ha relacionado a las mujeres con la "naturaleza" y a los hombres con la "cultura", perjudicando enormemente nuestra percepción de la historia y de las ciencias sociales 
en general (Moore 1991). Esta división dicotómica de la naturaleza humana ha supuesto que las actividades tradicionalmente asociadas con el sexo femenino, como gestar, parir, amamantar, cuidar los niños, cuidar a los enfermos y los ancianos, preparar las comidas, lavar la ropa,... sean consideradas como actividades humanas "naturales", entre otros motivos porque se las relacionan con necesidades biológicas inmediatas y, por tanto, irrelevantes social e históricamente, de ahí que queden totalmente excluidas del conocimiento histórico y prehistórico (Álvarez et alii 1992; Colomer et alii 1992; Sánchez Liranzo 1999; etc.).

Mientras, los hombres se han asociado con la "cultura", con la historia, con los grandes hechos, con la tecnología, con la ciencia, etc. Estas asociaciones tienen consecuencias muy nefastas para nuestro conocimiento prehistórico, ya que consolida al hombre como el protagonista principal y único del acontecer histórico, sosteniéndose así (consciente o inconsciente) que gracias a él y a sus actividades la sociedad y la civilización progresan.

\section{Disolución de los espacios femeninos y los espacios masculinos}

El pensamiento histórico tradicional tenía la necesidad de mantener espacios separados para los dos géneros. Sin embargo, actualmente pensamos que esta delimitación entre el espacio femenino y el espacio masculino no sólo está lejos de reflejar la realidad histórica o en nuestro caso prehistórica, sino que además colabora en la jerarquización de hombres y mujeres. El problema no es sólo que se encierre a las mujeres dentro del espacio "doméstico", sino que en el contexto actual el sistema de valores imperante apenas otorga importancia a lo que ocurre dentro de lo doméstico o privado.

Así, el ámbito público se relaciona con los espacios abiertos, accesibles como son foros, plazas, calles, parlamentos, fábricas,... que siempre han estado bajo la tutela masculina y que se han asociado con el dominio y el poder (Martínez 1996: 7).

Mientras, el ámbito privado o doméstico se define por su oposición al espacio público. En él se esconden aquellos aspectos de la vida que la historia tradicional considera vergonzosos (aspectos afectivos, corporales, personales) y que se oponen a los valores supuestamente masculinos (Ibídem: 7-8).

Los primeros estudios históricos de las mujeres fueron muy respetuosos con esta delimitación de espacios, de tal forma que hacer una historia de las mujeres era hacer una historia del espacio doméstico o privado.

En la actualidad, conscientes del androcentrismo de la historia (establecido gracias a la dicotomización y jerarquización de lo "femenino" y lo "masculino") las tendencias históricas próximas al feminismo tienden a eliminar y difuminar el límite entre ambos, viéndose directamente afectada la división entre el espacio doméstico y el espacio público. De hecho, no podemos seguir sosteniendo que los hombres se muevan única y exclusivamente dentro del ámbito público. ¿Acaso el hombre no tiene un espacio doméstico en el convive con las mujeres? ¿Por qué hemos de negarle al hombre un lugar en el espacio doméstico? Por otra parte, tampoco es cierto que las mujeres desarrollen su experiencia y vida sólo dentro del ámbito doméstico o privado, ¿acaso no son y han sido muy frecuentados por las mujeres lugares tan públicos como los mercados, las plazas, las calles, las iglesias, las fuentes,...? (Ibídem).

Desde esta reflexión pensamos que hay que comenzar a valorar en su justa medida lo ocultado y vincularlo con lo que siempre ha estado visible, es necesaria una relación dialéctica entre el espacio doméstico y el espacio público, que al final debe necesariamente desembocar en su disolución, al margen del protagonismo que unos y otras tengan en los distintos espacios. Ocultar lo doméstico o privado supone marginar no sólo a las mujeres, como ya hemos dicho, sino también ocultar aspectos básicos de los hombres que quedan escondidos en la "privacidad". 


\section{El concepto de relaciones de género}

Con el género se intentaba solventar la carencia de instrumentos conceptuales para analizar las diferencias sexuales de forma histórica, es decir se pretendía explicar cómo se construyen las identidades y los modos de vida de los hombres y de las mujeres. El género es una categoría de análisis histórico (Scott 1990) que nos ayuda a descubrir cómo los espacios, trabajos, valores y comportamientos de uno y otro género son un producto histórico.

Sin embargo, a pesar de que el género es una categoría muy útil para la construcción histórica, su definición y uso está lleno de dificultades en nuestra disciplina. Uno de los problemas más graves es que al ser definido como un elemento claramente ideológico difícilmente puede inferirse a partir de los productos arqueológicos encontrados.

Por otro lado, aunque se reivindica que los/as investigadores/as deben tener en cuenta las relaciones de género como parte de la estructura socio-económica, muchas veces se sigue aceptando que el género ha permanecido prácticamente inalterable desde los orígenes de la humanidad, al margen del contexto histórico. Olvidando así que las desigualdades sociales sean del tipo que sean son un producto histórico.

Otro problema importante que plantea este concepto es que ha sido abordado por algunos/as investigadores/as de forma dual y dicotómica, reproduciendo el sistema jerárquico que en principio pretendíamos rechazar; de hecho se habla de género "masculino" y "femenino" como si fueran grupos homogéneos y universales.

Desde nuestro punto de vista, no se trata de ver el género como una variable que tiene dos valores: masculino y femenino, sino como un sistema de relaciones, es decir que lo relevante no son los géneros en sí, sino las relaciones que se establece entre ellos y ellas (Izquierdo 1998). El género es una forma de referirnos a la organización social de las relaciones entre los sexos (Scott 1990).

\section{La vida cotidiana}

En la actualidad hay una necesidad de reivindicar la vida cotidian ${ }^{6}$ como el lugar en el que se desarrolla la experiencia histórica (y prehistórica) de los hombres y las mujeres. Sin embargo, a pesar de la importancia que tiene lo cotidiano, éste no puede investigarse al margen de la realidad social, política y económica. Así que estudiar la vida cotidiana no es hacer una descripción de las mujeres en el espacio doméstico, para ello es necesario estudiar lo privado y lo público en interacción y sus efectos en la vida cotidiana.

Es preocupante que los/as historiadores/as, pero sobre todo los/as arqueólogos/as, aún no hayamos demostrado apenas interés por la vida cotidiana como motor de la historia (Veloz 1984). En este sentido estamos de acuerdo con Agnes Heller (1972) cuando dice:

"La vida cotidiana no está fuera de la historia, sino en el centro del acaecer histórico: es la verdadera esencia de la sustancia social. Las grandes hazañas no cotidianas que se reseñan en los libros de historia arrancan de la vida cotidiana y vuelven a ella. Toda gran hazaña histórica concreta se hace particular e histórica precisamente por su posterior efecto en la cotidianeidad"

Todas estas propuestas no son más que un punto de partida para hacer una historia de las mujeres que contribuya al desarrollo de una historia (y prehistoria) más completa, más global, más universal y, por tanto, más próxima a la realidad histórica y prehistórica.

6. Hay quién señala que esto plantea el problema de que en realidad lo que se hace es ofrecer una visión microscópica de la historia, pero se olvida que la globalidad de la historia es un resultado que sin perspectiva hacia el interior del hecho cotidiano será imposible entender esa globalidad (Veloz 1984). 


\section{V. ¿PARA QUÉ LA HISTORIA DE LAS MUJERES?}

Nosotras pensamos que la utilidad de la historia de las mujeres es doble sirve a las mujeres, pero también sirve a la historia (y prehistoria) en general.

Como dijo Gerda Lerner, la historia es "una forma de mantener viva la memoria tanto colectiva como individual". De hecho, una de las razones por la que las mujeres han estado subordinadas al dominio del hombre durante milenios ha sido la carencia de historia propia, de un relato de su propia experiencia. Por tanto, necesitamos construir la historia, para recuperar nuestra memoria y reconocernos con las mujeres del pasado (Lerner 1990).

La inclusión de las mujeres como sujetos históricos de la historia, ahora sí más universal, no es sólo una cuestión moral oética, sino sobre todo una cuestión científica y epistemológica (Harding 1995), porque nos ayuda a pensar el mundo más rigurosa, objetiva y fundadamente. En este sentido hacemos nuestras las palabras de Isabel Morant cuando dice:

“(...) la Historia de las Mujeres es, así planteada, un camino obligado para la historia, porque lo hecho por las mujeres, su historia, no concierne sólo a media humanidad, sino a toda ella. Del mismo modo que la historia hecha por los hombres concierne igualmente a las mujeres y lo hecho por unas y por otros concierne a la historia general. Esto significa que, en la práctica, la construcción de un objeto histórico, bajo la rúbrica de Historia de las Mujeres por la orientación que se le daba estableciendo el carácter relacional de los sexos y considerando las diferencias como hechos sociales, debía tener efectos sobre el modo de hacer de los historiadores y sobre la historia. La Historia de las Mujeres, por otro lado, quedaba dentro de los planteamientos y de los métodos de la historia, desvelando eso sí temas e interrogantes nuevos para la historia social, política o económica, que implican, casi siempre, revisiones en el hacer del historiador" (1997: 16-17).

Estamos seguras/os que algunos de los planteamientos (feministas) aplicados a la arqueología y la prehistoria, contribuirán a un conocimiento más profundo de la realidad, porque nos ayudan a "desocultar" algunos prejuicios, estereotipos sexuales y ausencias de las mujeres que enmascaran el conocimiento histórico y prehistórico. Un conocimiento que estamos convencidas/os puede y debe contribuir a transformar de alguna forma la calidad de vida de todas las personas.

\section{BIBLIOGRAFÍA}

ÁLVAREZ, M.C.; BÓVEDA, M.J.; GÓMEZ, E.T.; VILASECA, X.I. (1992): “A muller: ¿sexe secundario?”, Reunión de Arqueología Teórica, Santiago de Compostela, 11-13 de noviembre, 1992.

BATE, L.F. (1998): El proceso de investigación en arqueología. Barcelona, Crítica.

COLOMER, E.; GILI, S.; GONZÁLEZ, P.; MONTÓN, S.; PICAZO, M.; RIHUETE, C.; RUIZ PARRA, M.; SANAHUJA, M.E.; SANZ, T. y TENAS I BUSQUETS, M.(1992): "Hacia una Arqueología Feminista", Reunión de Arqueología Teórica, Santiago de Compostela, 1-13 de noviembre, 1992.

HARDING, S. (1995): "Después del euroandrocentrismo: desafíos para la investigación feminista en el norte", en Martínez López (ed.): Feminismo, ciencia y transformación social. Colección Feminae. Universidad de Granada.

HELLER, A. (1972): Historia y Vida Cotidiana. Grijalbo.

IZQUIERDO, M. J. (1991): “Un marco teórico para las relaciones de sexo y de género”, en Luna (ed.) Mujeres y Sociedad. Nuevos Enfoques Teóricos y Metodológicos: 75-85. Seminario Interdisciplinar Mujeres y Sociedad, Madrid. (1998): El malestar en la desigualdad. Universidad de Valencia, Cátedra. 
LERNER, G. (1990): La creación del patriarcado. Barcelona, Crítica.

JULIANO, D. (1992): El juego de las astucias. Mujeres y construcción de modelos sociales alternativos. Madrid, Horas y Horas.

MARTÍNEZ, C. (1996): "Las mujeres y la historia", III Encuentro de Formación Feminista de Andalucía. Baeza, Instituto Andaluz de la Mujer.

MOORE, H.L.(1991): Antropología y feminismo. Universidad de Valencia, Cátedra.

MORENO, A.(1987): El arquetipo viril protagonista de la historia. Ejercicios de lectura no androcéntrica. Barcelona, Horas y horas.

MORANT, I. (1997): "Feminismo, Historia de las Mujeres e interpelaciones a la Historia", IV Encuentro Andaluz de Formación Feminista: El saber de las mujeres. Baeza, 25-28 Septiembre, 1997.

RAMOS, M.D.(1990): "Historiografía de la Mujer en la contemporaneidad: problemas y estado de la cuestión en Málaga", Primer Encuentro Interdisciplinar de Estudios de la Mujer en Andalucía, Granada.

SÁNCHEZ LIRANZO, O. (1999): "La prehistoria andaluza: una ciencia social que reproduce el discurso histórico androcéntrico", Revista Atlántico-Mediterránea de Prehistoria y Arqueología Social, vol. II, Universidad de Cádiz.

SÁNCHEZ LIRANZO, O. (2000): "La prehistoria en Andalucía durante el primer tercio del siglo XX. Las mujeres y el historicismo cultural", Arqueología Peninsular. História, Teoria e Prática. Porto. ADECAP.

SÁNCHEZ, A. (1991): "La masculinidad en el discurso científico: aspectos epistemológicos-ideológicos", en Luna (ed.) Mujeres y Sociedad. Nuevos enfoques teóricos y metodológicos. Madrid.

SCOTT, J.W. (1990): "El género: una categoría de análisis histórico", en Amelang y Nash (ed.) Historia y Género. Las mujeres en la Europa Moderna y Contemporánea. Valencia. Ed. Alfons el Magnánim. TABET, P. (1986): "La procréation comme travail", Côte Femmes. Approaches Ethnologiques. Paris, L'Hasmattan.

VELOZ, M. (1984): "La arqueología de la vida cotidiana: matices, historia y diferencias", Boletín de Antropología Americana 10: 5-21. México.

VERA FERNÁNDEZ, A.; SÁNCHEZLIRANZO, O. (2000): "La historiografía prehistórica como conciencia histórica", Arqueología Peninsular. História, Teoria e Prática. Porto. ADECAP. 